\title{
Influence of the STA boundary conditions on thermal decomposition of thermoplastic polymers
}

\author{
$\underline{\text { David LÁZARO }}^{*}$, Mariano LÁZARO ${ }^{1}$, Alain ALONSO ${ }^{1}$, Pedro LÁZARO ${ }^{1}$, Daniel \\ ALVEAR ${ }^{1}$ \\ ${ }^{1}$ GIDAI Group, University of Cantabria, Avda de Los Castros, s/n. 39005 Santander, Spain
}

The analysis of polymer chemical decomposition is often highly dependent on the test conditions. In fact, thermal analysis tends to be far more sensitive to instrumental parameters than other branches of chemical analysis. Some of the boundary conditions in thermal analysis have been widely studied in the literature, such as the heating rate or the atmosphere. However, the influence of the sample mass, the gas flow or the use of lid have not been enough studied for thermoplastic polymers.

The aim of this paper is to analyse how the experimental boundary conditions of the Simultaneous Thermal Analysis apparatus affect the thermal decomposition of thermoplastic polymers. To do so a set of 35 experimental tests have been performed including variation of the sample mass, gas flow rate, heating rate, atmosphere and the use of lid in the crucible. Results enable us to analyse the influence in the mass loss and in the energy release or absorbed in the thermal decomposition of thermoplastic polymers, showing the impact of each boundary condition over the thermal decomposition. A comprehensive analysis of the thermal decomposition behaviour of the PVC and LLDPE by considering the influence of all the boundary conditions of the STA is covered. It is especially remarkable the influence of gas flow in the oxidative reactions, and of heating rate in the chemical reactions that thermoplastics polymers undergo in their decomposition. Additionally, sample mass comparison shows only deviation in the oxidative reactions, and do not show deviation for the non-oxidative reactions. That seems to show a higher effect on the results because of the energy release in the decomposition reactions than because of the thermal lag due to heat transfer on the sample, as it is usually thought.

Keywords TG profiles, DSC profiles, LLDPE, PVC, thermal decomposition

*corresponding author: email: lazarod@unican.es; Telf.+34 942201826; https://orcid.org/0000-0002-8150-4838

\section{INTRODUCTION}

Thermal decomposition of polymers is a complex process due to several mechanisms, such as end-chain and random-chain scissions, chain stripping and cross-linking, as well as their own chemical formulation, which is different for each polymer. Moreover, the chemical reactions produced during the thermal decomposition usually rely on the boundary conditions. In fact, thermal analysis tends to be far more sensitive to instrumental parameters than other branches of chemical analysis [1].

The Simultaneous Thermal Analysis (STA) apparatus combines Thermogravimetry (TG) and Differential Scanning Calorimetry (DSC) in a single test to measure the mass change and the heat flow as function of temperature. The tests performed by STA are defined by the boundary conditions that configure the experiments. Some boundary conditions, such as heating rate and the atmosphere, have been widely studied and their influence in the mass loss can be found in the literature [2,3]. However, others issues, like sample mass, gas flow or the employment of lid, have not received considerable attention, neither the influence of the boundary conditions in the energy release or absorbed.

As far as the initial mass is concerned, standards include several recommendations. The ASTM E1131 - 08 [4] recommends sample mass of 10 to $30 \mathrm{mg}$ for the TG. ISO standards recommends an initial mass between $10 \mathrm{mg}$ and $100 \mathrm{mg}$ for the TG [5] and between 2 and $40 \mathrm{mg}$ for the DSC [6]. Additionally, in [7] NETZSCH performed their own tests by using samples between 9 and $11 \mathrm{mg}$, to create a computer-assisted database and to identify polymers by means of DSC, TG and STA. Nevertheless, sample mass lower than $10 \mathrm{mg}$ has been widely used by scientific community to perform TG analysis of different kinds of materials [8-13]. In fact, the Kinetics Committee of the International Confederation for Thermal Analysis and Calorimetry (ICTAC) presented an equation (Eq. 1) to 
estimate the temperature gradient inside the sample. ICTAC recommends in order to minimize the errors in the evaluation of the kinetic parameters, to select the sample thickness and heating (cooling) rate so that the temperature gradient does not exceed a few degrees.

$$
\Delta T=\frac{\beta h^{2}}{4 \lambda}
$$

Assuming that a sample is shaped as a flat layer of the height $h$, that $\lambda$ is the sample thermal diffusivity, and $\beta$ is the heating rate. According to the equation (1), as lower is the height (and therefore the mass of the sample), the lower is the temperature gradient.

Related to the influence of the gas flow on the STA experimental test, ASTM E1131 - 08 [4] recommends a flow rate of $50 \mathrm{ml} \cdot \mathrm{min}^{-1}$ for thermoplastic, ISO 11358-1 [5] allows flow rates between 10 and $150 \mathrm{ml} \cdot \mathrm{min}^{-1}$, and ISO 11357-1 [6] flow rates between 10 and $150 \mathrm{ml} \cdot \mathrm{min}^{-1}$. A large amount of works set different values of the gas flow rate, like $50 \mathrm{ml} \cdot \mathrm{min}^{-1}$ [14], $60 \mathrm{ml} \cdot \mathrm{min}^{-1}[10,15,16], 100$ and $80 \mathrm{ml} \cdot \mathrm{min}^{-1}$ [17] or $200 \mathrm{ml} \cdot \mathrm{min}^{-1}$ [18]. Furthermore, some studies do not include the gas flow value employed in the STA tests [19, 20].

Abu-Bakar and Moinuddin [21] studied the influence of the sample mass and the gas flow in the TG for PMMA and pine materials, obtaining slight differences in the PMMA sample. The influence of the sample mass and the gas flow in the thermal degradation of the polypropylene under an air atmosphere was also studied by Stawski [22]. Results showed a clear dependence of the thermal decomposition on the initial mass of the samples. By the contrast, any influence of the gas flow on the decomposition was appreciated. Nevertheless, these studies do not include the influence of the sample mass neither the gas flow in the DSC, while the analysis of the reaction energies can give much more information about the effect of those boundary conditions in the thermal decomposition of the materials.

The influence of heating rate on the mass loss of PE, PET, PVC, PS, cellulose, hemicellulose, lignin, pectin and starch samples of around $1.5 \mathrm{~g}$ is analyzed on [23]. They used a macro-TG and claimed that samples delay their decomposition and the main mass loss rate peak temperature by increasing the heating rate, except for lignin. Nevertheless, the sample mass used in the macro-TG will influence the behaviour with respect to TG analysis. It can be seen in the effect of heating rate variation on PVC decomposition in the study [2], where oxidative reactions were more affected by the heating rate than in the PVC analyzed in [23] with larger mass samples. The analysis of the DSC is not included in these studies, and it could give more information about the oxidative reactions.

Therefore, a study of sample mass effect on STA is highly likely to determine whether tests should be performed following the standards recommendations, or a lower initial mass should be used. On the other hand, since only two references have been found regarding gas flow effect on TG, it seems necessary a better understanding of its effect.

The employment of a lid to cover the samples is recommended by the normative ASTM $1269-11$ [25] to determine the specific heat capacity. In addition, the use of a lid permits to measure thermal decomposition in selfgenerated atmosphere and to obtain a more homogeneous heat distribution within the crucible. The influence of the use of pierced lid in the DSC has been studied by Wolfinger et al. [25]. The conclusions showed the requirement of crucibles without a lid when the reaction conditions require a fast gas exchange between the sample and the surroundings. It is remarked the influence of the heat flux rate of radiation when sample and reference have different emissivity. That effect occurs when lid is not used. Nevertheless, this study is done under an inert atmosphere which means that none effect of the lid into the atmosphere over the sample can be studied, to do this, oxidative atmosphere should be used.

We selected poly (vinyl chloride) (PVC) and the linear low density polyethylene (LLDPE) for this study since they are two of the most used thermoplastic polymers. Additionally, they have a large number of different kinds of chemical reactions, hence the results could be extrapolated to other materials. In addition, it will allow a better understanding of how each single boundary condition affects the behaviour of the decomposition reactions, and it will facilitate the selection of the different boundary conditions. To sum up, our analysis includes the full study of the STA sample mass, the gas flow rate, the use of lid in the crucible, the atmosphere of the sample and the heating rate influence in the TG and in the DSC. This allow to better understand the effect of the different boundary conditions in the decomposition reactions, as not only the mass, but also the decomposition reactions energies results 
are included in the study. And it also allows a completely understood of the thermal decomposition behaviour of the PVC and LLDPE as a full analysis of 35 different tests were performed.

\section{EXPERIMENTAL TESTS}

The Netzsch STA 449 F3 was used to perform the experimental campaign, which allowed to test in a range of temperature between 30 and $1500{ }^{\circ} \mathrm{C}$ in oxidative or inert atmosphere. The temperature and balance resolutions are $0.001 \mathrm{~K}$ and $0.1 \mu \mathrm{g}$ over the entire weighing range, respectively. The DSC enthalpy accuracy is $\pm 2 \%$ for most materials.

$\operatorname{PVC}\left(\left(\mathrm{C}_{2} \mathrm{H}_{3} \mathrm{Cl}\right)_{n}\right)$ is a polymer prepared from Vinyl Chloride Monomer (VCM). Thermal decomposition of PVC is substantially a two-stage process. While the first step $\left(227-377^{\circ} \mathrm{C}\right)$ mainly involves the progressive dehydrochlorination of the polymer that consists on the elimination of $\mathrm{HCl}$, at temperatures over approximately 377 ${ }^{\circ} \mathrm{C}$, a second mass loss can be observed which corresponds to the pyrolysis of the polyene sequences formed during the previous reaction. Some evaporation of plasticizer may be also observed in the first step [9] and the loss of $\mathrm{HCl}$ produces a mass loss of approximately $65 \%$. The total mass loss in this chemical reaction is slightly higher than the stoichiometric quantity of $\mathrm{HCl}$ in the PVC. This may be produced by the formation of some aromatic hydrocarbons, mainly benzene.

Linear low-density polyethylene (LLDPE) is made of the copolymerization of ethylene and a comonomer. Typically, comonomers used for the polymerization of LLDPE are butene, hexene, and octene. The main polymer chain of LLDPE is composed of long strings of repeating ethylene molecules; the comonomer forms short side chains which are linked to the main chain. LLDPE consists of long linear molecules with short side chain branches (SCB). SCB length is a function of the comonomer type employed [26].

In order to analyse the variation of the thermal decomposition behaviour with the boundary conditions, a reference case has been defined, and then, in the rest of the cases vary only one of the boundary conditions. Reference tests, denoted as Case 1 for PVC and Case 19 for LLDPE, were considered in air atmosphere without lid with a gas flow rate of $40 \mathrm{ml} \cdot \mathrm{min}^{-1}$ and the sample was heated from 30 to $800^{\circ} \mathrm{C}$ for PVC and 30 to $650{ }^{\circ} \mathrm{C}$ for LLDPE under a heating rate of $10 \mathrm{~K} \cdot \mathrm{min}^{-1}$. The initial masses considered in that reference case were $12.184 \mathrm{mg}$ and $12.139 \mathrm{mg}$ for the PVC and the LLDPE, respectively. A total of 18 tests were carried out for PVC and 17 for LLDPE, varying only one boundary condition in each experimental test (Table 1). These variated boundary conditions are remarked in italic for each test in the Table 1.

Table 1 Cases for the STA analysis.

\begin{tabular}{|c|c|c|c|c|c|c|}
\hline Test (Case) & Material & Atmosphere & $\begin{array}{l}\text { Initial Mass } \\
\text { /mg }\end{array}$ & Lid & $\begin{array}{c}\text { T.min } /{ }^{\circ} \mathrm{C}-\mathrm{HR} / \mathrm{K} \cdot \mathrm{min}^{-1} \cdot- \\
\mathrm{T} \cdot \max /{ }^{\circ} \mathrm{C}\end{array}$ & $\begin{array}{l}\text { Gas Flow } \\
/ \mathrm{ml} \cdot \mathrm{min}^{-1}\end{array}$ \\
\hline 1 & PVC & Air & 12.184 & No & $30-10.0-800$ & 40 \\
\hline 2 & PVC & Air & 12.212 & No & $30-10.0-800$ & 40 \\
\hline 3 & PVC & Air & 12.179 & No & $30-10.0-800$ & 40 \\
\hline 4 & PVC & Air & 20.736 & No & $30-10.0-800$ & 40 \\
\hline 5 & PVC & Air & 4.763 & No & $30-10.0-800$ & 40 \\
\hline 6 & PVC & Air & 14.594 & No & $30-10.0-800$ & 40 \\
\hline 7 & PVC & Air & 6.576 & No & $30-10.0-800$ & 40 \\
\hline 8 & PVC & Air & 18.435 & No & $30-10.0-800$ & 40 \\
\hline 9 & PVC & Air & 12.251 & No & $30-10.0-800$ & 150 \\
\hline 10 & PVC & Air & 12.174 & No & $30-10.0-800$ & 50 \\
\hline 11 & PVC & Air & 12.195 & No & $30-10.0-800$ & 10 \\
\hline 12 & PVC & Air & 9.349 & Yes & $30-10.0-800$ & 40 \\
\hline 13 & PVC & $N_{2}$ & 13.079 & No & $30-10.0-800$ & 40 \\
\hline 14 & PVC & $\mathrm{N}_{2}$ & 12.639 & Yes & $30-10.0-800$ & 40 \\
\hline 15 & PVC & $5 \% \mathrm{O}_{2}-95 \% \mathrm{~N}_{2}$ & 12.612 & No & $30-10.0-800$ & $10\left(\mathrm{O}_{2}\right)-30\left(\mathrm{~N}_{2}\right)$ \\
\hline
\end{tabular}




$\begin{array}{llccccc}16 & \text { PVC } & 10 \% \mathrm{O}_{2}-90 \% N_{2} & 13.589 & \text { No } & 30-10.0-800 & 20\left(O_{2}\right)-20\left(N_{2}\right) \\ 17 & \text { PVC } & \text { Air } & 13.057 & \text { No } & 30-5.0-800 & 40 \\ 18 & \text { PVC } & \text { Air } & 11.926 & \text { No } & 30-20.0-800 & 40 \\ 19 & \text { LLDPE } & \text { Air } & 12.139 & \text { No } & 30-10.0-650 & 40 \\ 20 & \text { LLDPE } & \text { Air } & 11.849 & \text { No } & 30-10.0-650 & 40 \\ 21 & \text { LLDPE } & \text { Air } & 12.863 & \text { No } & 30-10.0-650 & 40 \\ 22 & \text { LLDPE } & \text { Air } & 3.585 & \text { No } & 30-10.0-650 & 40 \\ 23 & \text { LLDPE } & \text { Air } & 1.465 & \text { No } & 30-10.0-650 & 40 \\ 24 & \text { LLDPE } & N_{2} & 9.924 & \text { No } & 30-10.0-600 & 40 \\ 25 & \text { LLDPE } & N_{2} & 8.666 & \text { No } & 30-5.0-600 & 40 \\ 26 & \text { LLDPE } & N_{2} & 9.744 & \text { No } & 30-2.0-600 & 40 \\ 27 & \text { LLDPE } & N_{2} & 22.106 & Y e s & 50-10.0-650 & 40 \\ 28 & \text { LLDPE } & \text { Air } & 11.625 & Y e s & 50-10.0-650 & 40 \\ 29 & \text { LLDPE } & 5 \% \mathrm{O}_{2}-95 \% \mathrm{~N}_{2} & 15.324 & \text { No } & 30-10.0-650 & 10\left(O_{2}\right)-30\left(N_{2}\right) \\ 30 & \text { LLDPE } & 10 \% \mathrm{O}_{2}-90 \% \mathrm{~N}_{2} & 12.96 & \text { No } & 30-10.0-650 & 30\left(O_{2}\right)-30\left(N_{2}\right) \\ 31 & \text { LLDPE } & \text { Air } & 12.893 & \text { No } & 30-10.0-650 & 100 \\ 32 & \text { LLDPE } & \text { Air } & 11.961 & \text { No } & 30-10.0-650 & 60 \\ 33 & \text { LLDPE } & \text { Air } & 12.518 & \text { No } & 30-10.0-650 & 10 \\ 34 & \text { LLDPE } & \text { Air } & 11.919 & \text { No } & 30-20.0-600 & 40 \\ 35 & \text { LLDPE } & \text { Air } & 12.186 & \text { No } & 30-5.0-600 & 40\end{array}$

\subsection{Analysis of repeatability}

Reference case test was performed three times to analyse the repeatability of the result. Figures 1 and 2 show a very good repeatability in the PVC tests. Cases 1 to 3 have the same boundary conditions with a mass of $12.184 \mathrm{mg} \pm$ $0.23 \%$, and there is only a slightly difference in the last DSC peak. It can be seen in the Figures 1 and 2 the endothermic dehydrochlorination reaction at around $292{ }^{\circ} \mathrm{C}$, and then several exothermic reactions above $400{ }^{\circ} \mathrm{C}$. First dehydrochlorination reaction is independent of the atmosphere, while the exothermic reactions depend on the atmosphere.

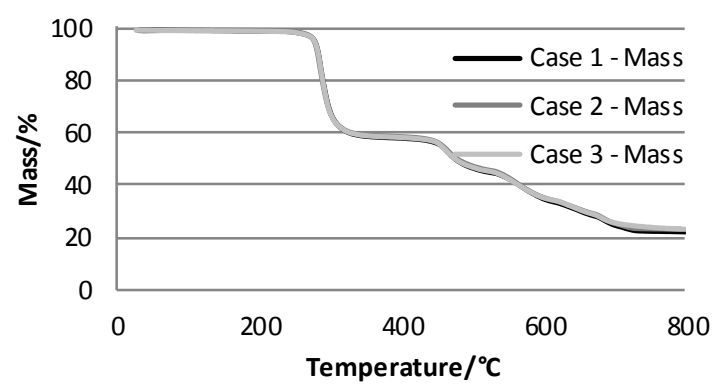

Fig. 1 Repeatability on TG curves for PVC.

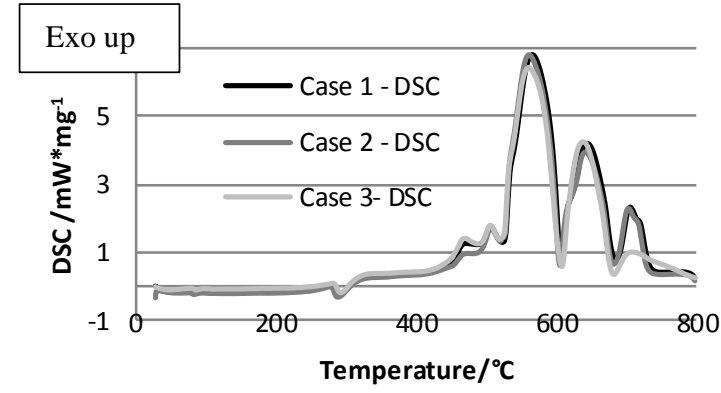

Fig. 2 Repeatability on DSC curves for PVC.

For the LLDPE, cases 19 to 21 have the same boundary conditions with a mass of $12.139 \mathrm{mg} \pm 6.00 \%$. Figures 3 and 4 show a very good repeatability. At around $129^{\circ} \mathrm{C}$, LLDPE has the melting reaction (Figure 4), this endothermic reaction is followed by several exothermic oxidative reactions above $250^{\circ} \mathrm{C}$. 


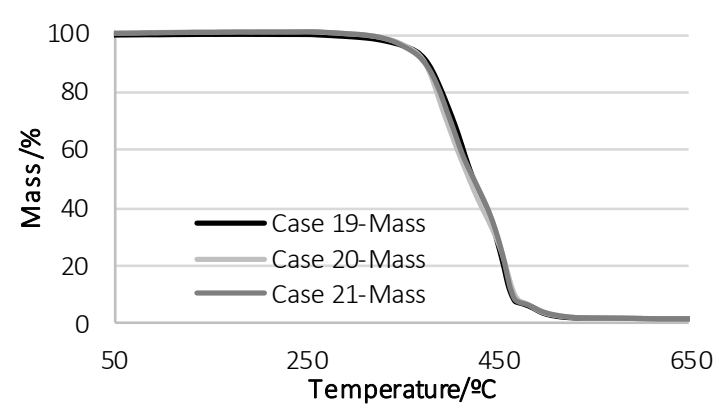

Fig. 3 Repeatability on TG curves for LLDPE.

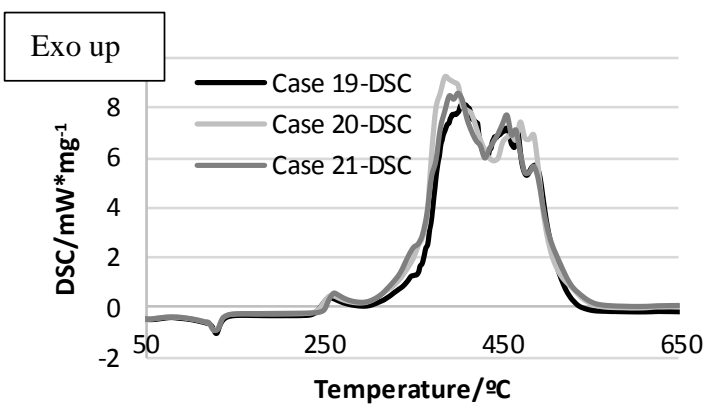

Fig. 4 Repeatability on DSC curves for LLDPE.

\section{RESULTS AND DISCUSSION}

\subsection{Analysis of sample mass}

To study the influence of the variation of the initial mass, 6 cases were compared for the PVC and 3 cases for the LLDPE. Figure 5 gathers the initial mass of the samples and the variation versus the Case 1, which is the reference case considered for the PVC. As it can be seen in Figure 5, cases 5 and 7 have less than $10 \mathrm{mg}$, while the other samples have more that $10 \mathrm{mg}$.

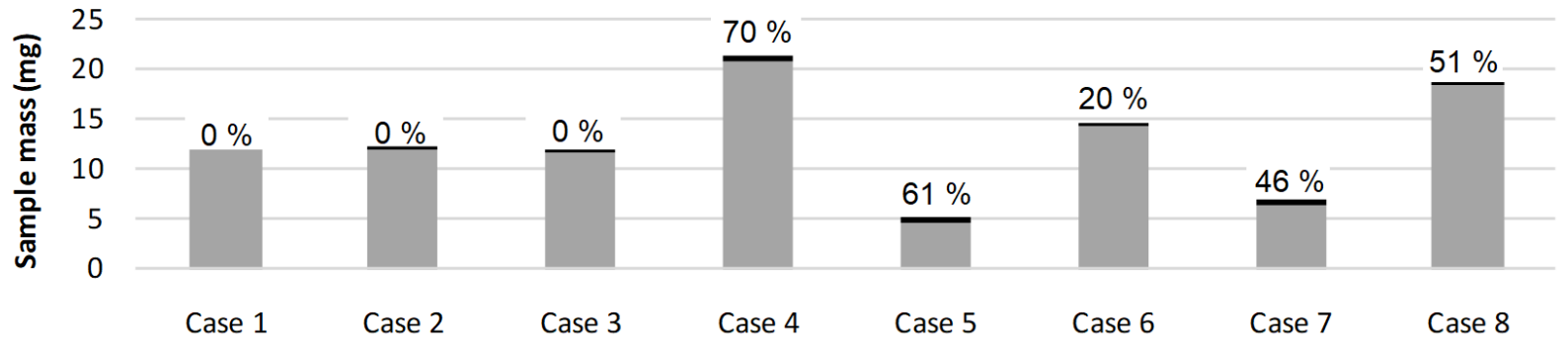

Fig. 5 Mass samples variation and relative variation for PVC analysis.

Figure 6 shows that mass loss tended to be similar in all the cases except in number 5 and 7, which correspond to the samples with a mass of 4.763 and $6.576 \mathrm{mg}$. Until oxidation reactions began, the TG and DSC curves were similar in all cases, that means that dehydrochlorination reaction is independent of the sample mass.

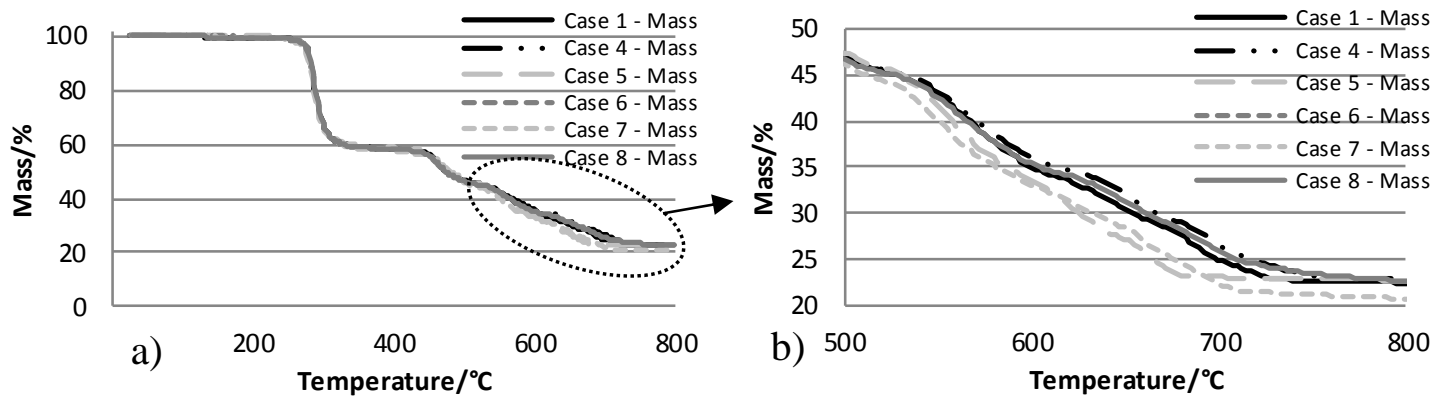

Fig. 6 Sample mass influence on PVC TG curve (a-total results, b-zone of interest).

The heat released per unit of mass in the first oxidative reaction of the PVC, R1, was higher in cases 5 and 7 (Figure 7), and they lost more mass than other cases. This oxidative reaction took place between 500 and $600{ }^{\circ} \mathrm{C}$. 
We can also observe that oxidative reaction peaks for samples with a mass lower than $10 \mathrm{mg}$ occurred at lower temperatures.
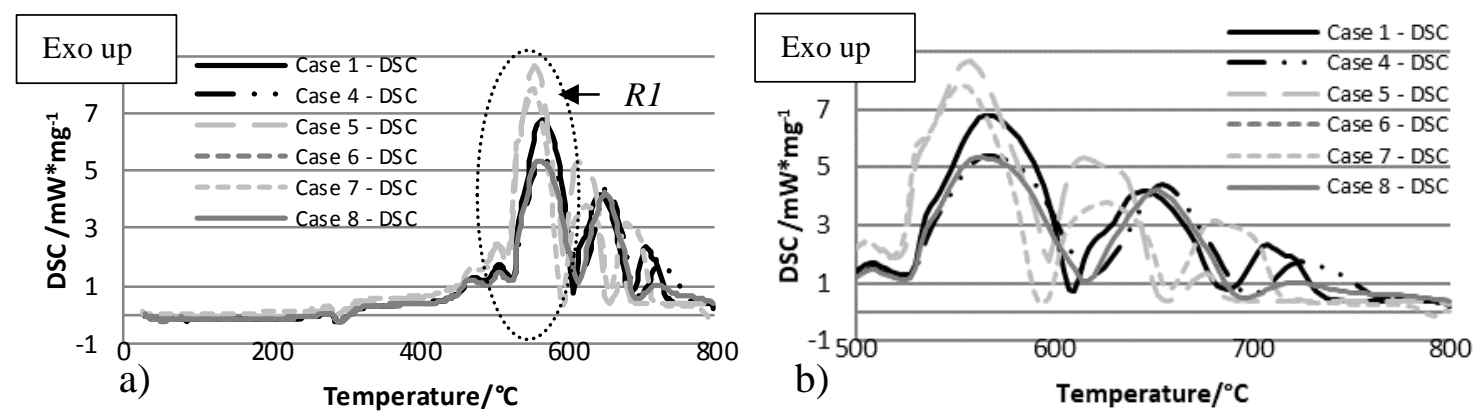

Fig. 7 Sample mass influence on PVC DSC curve (a-total results, b-zone of interest).

Next Figure 8 gathers the cases employed to analyze the influence of the sample mass for the LLDPE and the variation of its mass considering the case 19 as a reference. For the LLDPE cases 22 and 23 have an initial mass lower than $10 \mathrm{mg}$.

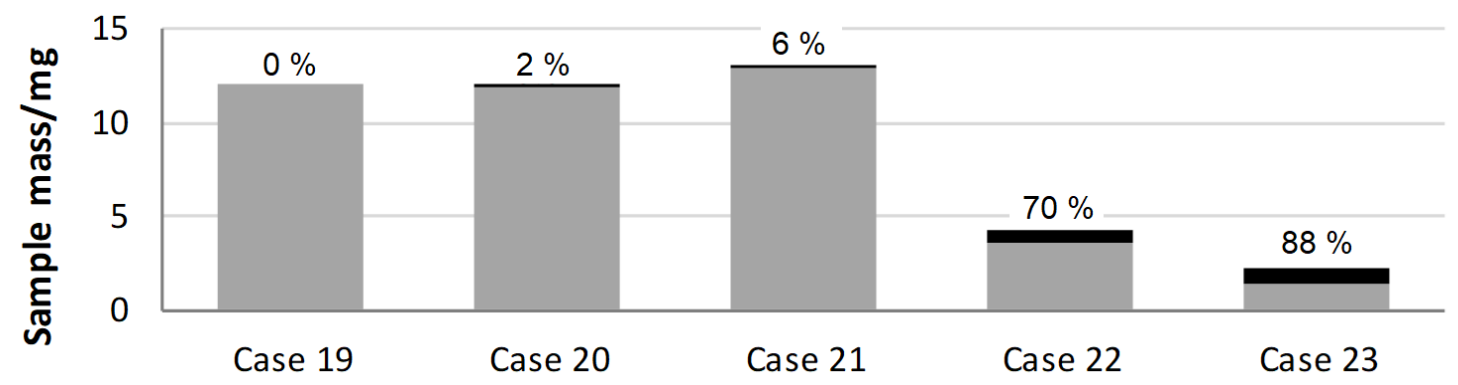

Fig. 8 Mass samples variation and relative variation for LLDPE analysis.

Figure 9 shows how the decomposition temperature of the LLDPE decrease by decreasing the mass of the sample. Additionally, it can be observed in the slope of the TG curves that the oxidative reactions are more defined with the lower sample mass. According to the energy (Figure 10), it can be seen that, as in the PVC, the lower the sample mass, the higher heat release per unit of mass.

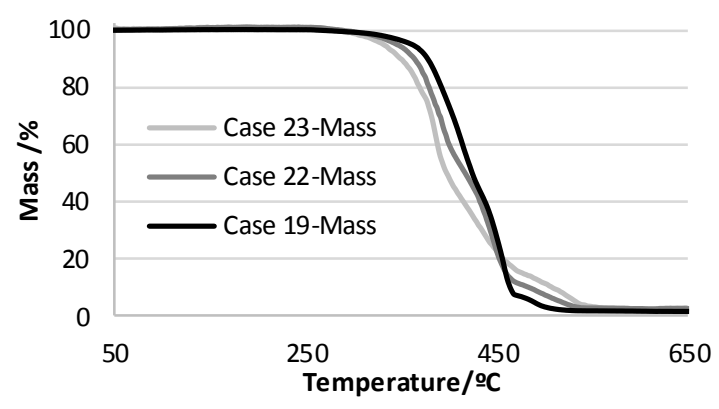

Fig. 9 Sample mass influence on LLDPE TG curve.

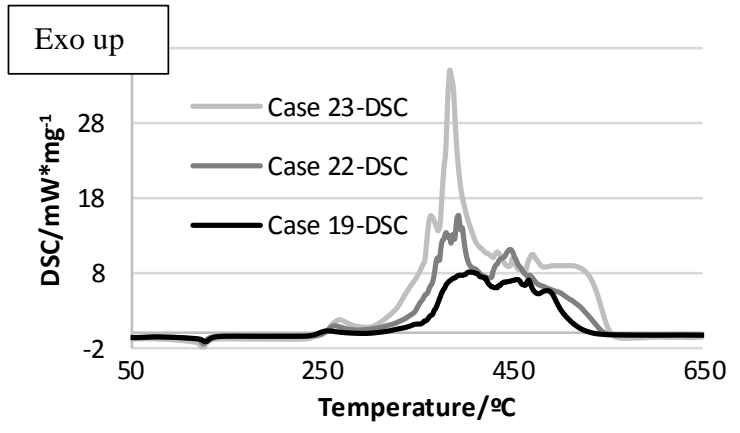

Fig. 10 Sample mass influence on LLDPE DSC curve. 
On the whole, the mass is related with the thermal inertia of the sample, and it affects directly the heating of the sample. Nevertheless, in this study we modified sample masses from 4.763 to $20.736 \mathrm{mg}$ for PVC and from 1.465 to $12.863 \mathrm{mg}$ for LLDPE, and variation in the results have been only found in the oxidative reactions. This could have more relationship with the availability of oxygen by all the mass of the sample than with the thermal inertia. The thinnest samples had much more percent of it mass in contact with the atmosphere, and therefore, the most part of the sample decomposed under oxygen atmosphere.

\subsection{Analysis of gas flow}

To analyze the gas flow influence in the thermal decomposition of PVC in STA tests, we used gas flows of 10, 40, 50 and $150 \mathrm{ml} \cdot \mathrm{min}^{-1}$ (Cases 11, 1, 10 and 9, respectively). As it is shown in Figures 11 and 12, the dehydrochlorination reaction of the PVC was not affected by the inlet gas flow, neither in the mass loss nor the heat absorbed.

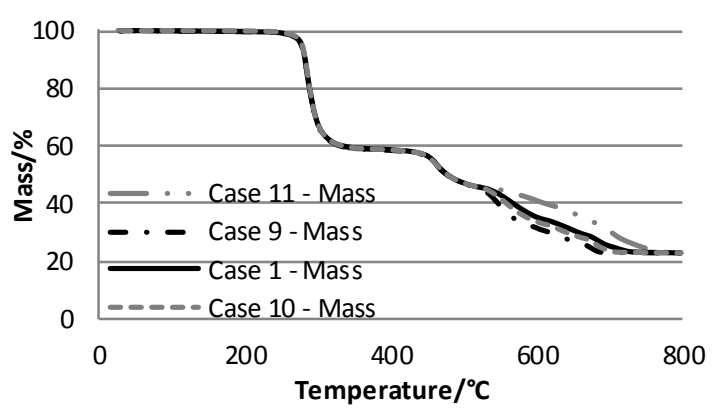

Fig. 11 Gas flow influence on PVC TG curve.

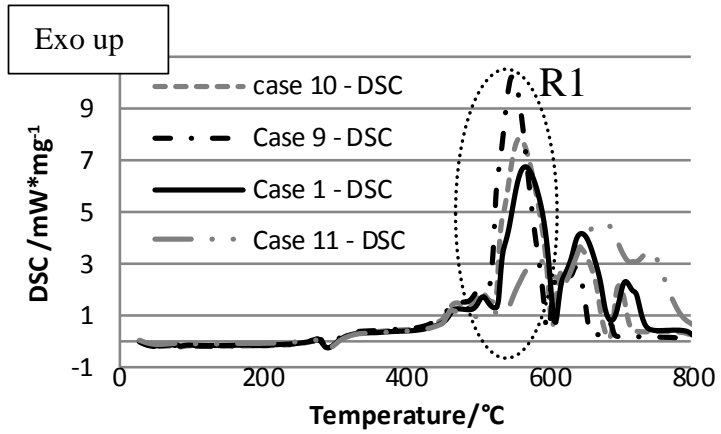

Fig. 12 Gas flow influence on PVC DSC curve.

The differences in the mass loss and in the DSC curves began with the oxidative reactions. On the one hand, the higher the gas flow rate, the higher the first oxidation reaction R1 peak (case 9), and on the other hand, when gas flow is higher, the mass loss in R1 is obtained faster and larger amount of heat per unit of mass is released in R1. In addition, we can observe that for the lower gas flow, case 11, the oxidation reactions are delayed and overlapped, since the oxidative chemical reactions had less air available.

Same behaviour happened in the LLDPE test results. Figures 13 and 14 show TG and DSC results for the gas flows of 10, 40, 60 and $100 \mathrm{ml} \cdot \mathrm{min}^{-1}$ (cases 33, 19, 32 and 31 respectively). It can be seen in Figure 14 that melting is not affected by the gas flow. STA results obtained under gas flows of 60 and $100 \mathrm{ml} \cdot \mathrm{min}^{-1}$, are quite similar. Decomposition reactions of case 33 happens at higher temperatures, and we can see in the DSC that these decomposition reactions begin as exothermic ones, but there is an endothermic peak around the $450{ }^{\circ} \mathrm{C}$. The rest of cases have exothermic reactions. 


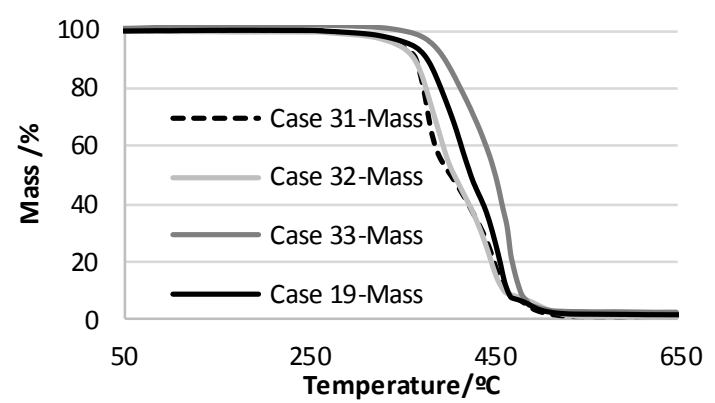

Fig. 13 Gas flow influence on LLDPE TG curve.

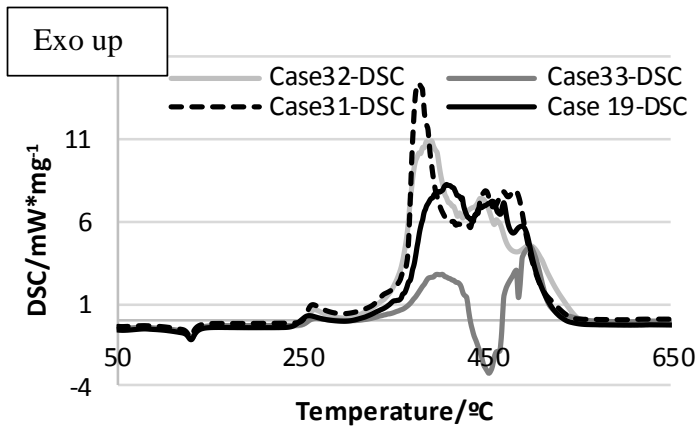

Fig. 14 Gas flow influence on LLDPE DSC curve.

It can be observed that the variation of the gas flow only affects to the oxidative reactions. This is related with the oxygen availability by the sample to decompose. The gas flow will determine the kind of combustion that undergoes the sample. Cases 32 and 31 have similar results, although gas flow varies from 60 to $100 \mathrm{ml} \cdot \mathrm{min}^{-1}$. This is because at these gas flows the combustion reactions have an excess of oxygen, hence combustion is limited by the combustible. Nevertheless, when a gas flow of $10 \mathrm{ml}^{*} \mathrm{~min}^{-1}$ is selected, the combustion is underventilated, and there is a lack of oxygen that makes that during decomposition an endothermic reaction takes place (Figure 14).

\subsection{Analysis of the effect of the lid}

Several STA tests with and without lid under inert and oxidative atmosphere were performed to study the effect of the lid in the analysis of the thermal decomposition of PVC and LLDPE. The use of both atmospheres allows to discriminate whether the effect of the lid is because of the atmosphere or caused by another physical effect.

Figures 15 and 16 show the TG and DSC results for PVC samples with and without lid under an oxidative (air with $21 \%$ of oxygen) and inert (nitrogen) atmosphere. Under air atmosphere, the sample without lid (case 1) releases more heat and it has a higher mass loss rate in the first reaction of oxidation. This might well be due to a lower availability of oxygen in the sample with lid (case 12). In this case, the gases released during the thermal decomposition pushes the air out of the crucible, decreasing oxygen concentration available to the oxidation of the sample.

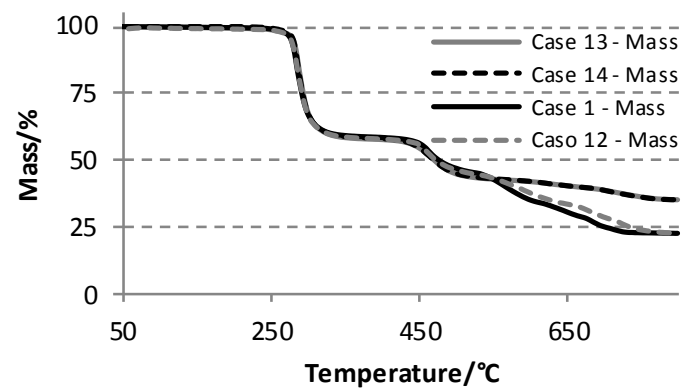

Fig. 15 Lid influence on PVC TG curve.

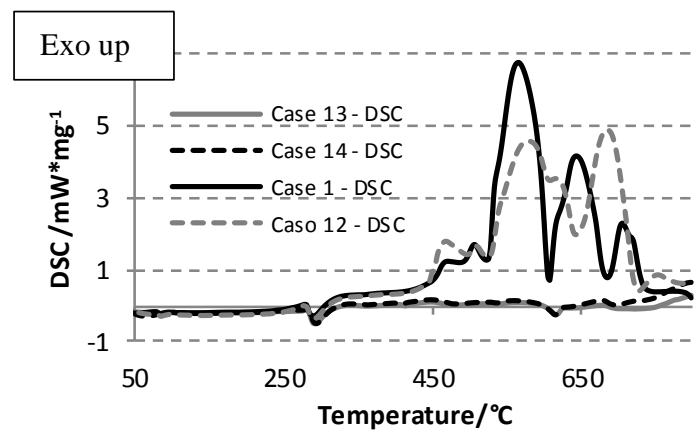

Fig. 16 Lid influence on PVC DSC curve.

The dehydrochlorination reactions are quite similar, so the atmosphere does not have any influence on these reactions. We can observe some differences on the DSC curve at high temperatures, which can be related to the radiation effect that affect the sample at high temperatures [25].

The effect of the lid is much obvious in LLDPE results. As it can be seen in the Figure 17 for TG and Figure 18 for DSC, under the nitrogen atmosphere there is not a significant effect in the mass loss, nevertheless, as happens 
in the PVC, there is a variation in the DSC curve at high temperatures. The use of a lid under an oxidative atmosphere (Case 28) delays decomposition and decreases the heat released per unit of mass by the sample.

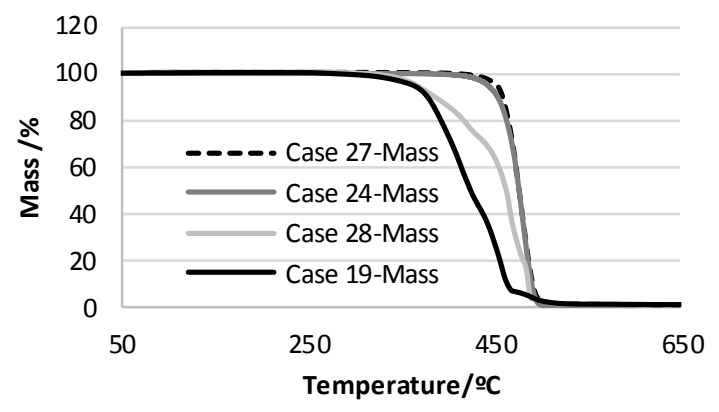

Fig. 17 Lid influence on LLDPE TG curve.

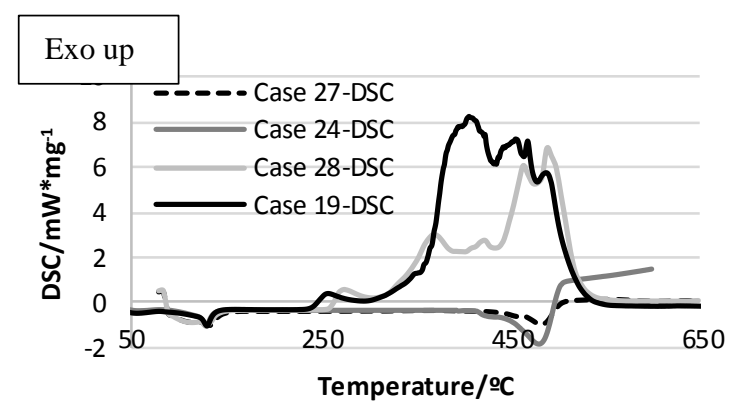

Fig. 18 Lid influence on LLDPE DSC curve.

\subsection{Analysis of the atmosphere}

In some cases, it is interesting to study the thermal decomposition under limited oxygen atmospheres. Figures 19 and 20 show the PVC mass loss and DSC under different atmospheres with oxygen concentrations of 21, 10, 5 and $0 \%$ (cases 1, 16, 15 and 13, respectively), and the Figures 21 and 22 show the LLDPE mass loss and DSC under different atmospheres with oxygen concentrations of 21, 10, 5 and $0 \%$ (cases 19,30, 29 and 24, respectively).

The decomposition under the atmosphere with $21 \%$ of $\mathrm{O}_{2}$ (case 1) has the highest mass loss in the first oxidative reaction. It also has the highest heat release per unit of mass (Figures 19 and 20), since the gases released in the polymer decomposition have more oxygen available to react. The heat released, in this first oxidative reaction, increases the polymer temperature allowing more decomposition and releasing more gases from the sample.

When lowest oxygen tests are performed, less quantity of gases are released from the decomposition of the polymer. This makes that the first oxygen reaction occurs at higher temperatures and releases less heat of reaction. When the second reaction takes place, the polymer sample is less degraded, therefore more gases can release to react with the oxygen available. If there is not oxygen available for the oxidation reactions, the decomposition reactions that take place up to $500{ }^{\circ} \mathrm{C}$ are endothermics (Figure 20) and PVC losses less mass in its decomposition (Figure 19).

Figure 20 also shows how the oxidative reactions are better defined with higher amount of oxygen. Figure 19 shows the same mass loss in the dehydrochlorination reaction for all the cases, as this reaction is independent of the atmosphere.

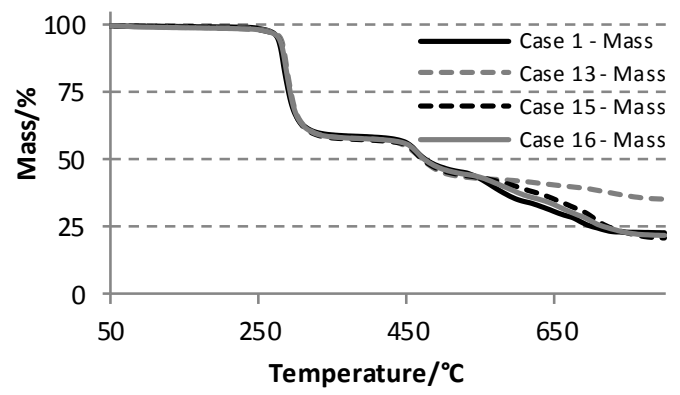

Fig. 19 Atmosphere influence on PVC TG curve.

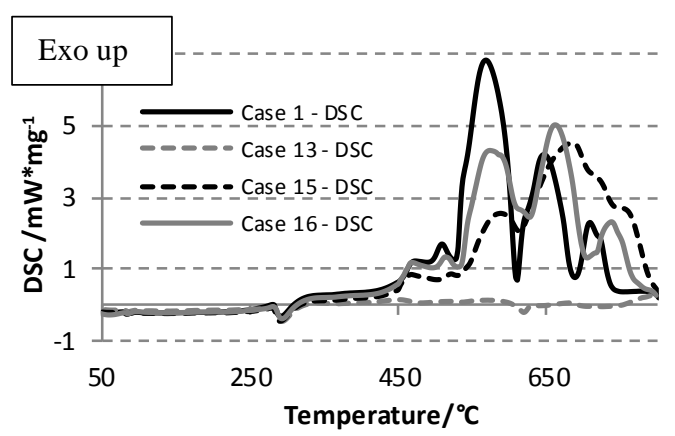

Fig. 20 Atmosphere influence on PVC DSC curve.

Figures 21 and 22 show the comparison of the mass loss and DSC for samples under atmospheres with an oxygen concentration of $21,10,5$ and $0 \%$ of Oxygen. Figures 21 and 22 show that the higher the oxygen 
concentration is, the lower is the decomposition reaction temperature and the higher is the amount of heat released per unit of mass during decomposition.

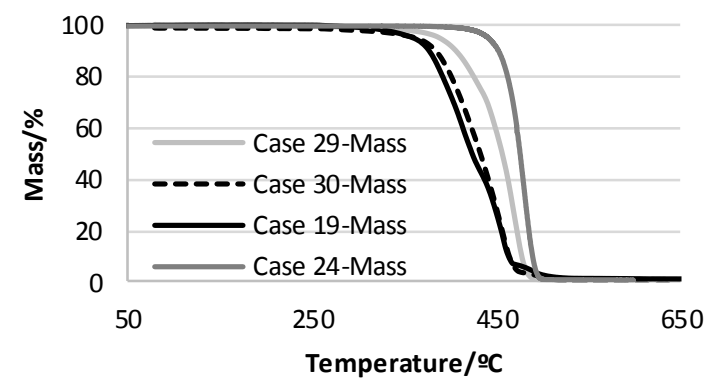

Fig. 21 Atmosphere influence on LLDPE TG curve.

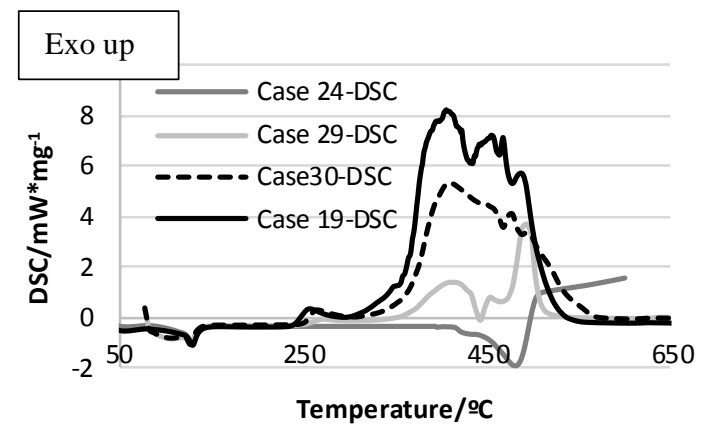

Fig. 22 Atmosphere influence on LLDPE DSC curve.

\subsection{Analysis of the heating rate}

The variation of the heating rate of the TG tests is widely used in scientific community for different goals. The use of lower heating rates allows separating some decomposition reactions which will be shown superposed at higher heating rates. The heating rate will also affect the temperatures at which the different reactions occurs. The lower the heating rate is, the lower is the temperature at which the reactions occur.

Figures 23 and 24 show the STA results of the PVC samples for the heating rates of 5, 10 and $20 \mathrm{~K} \cdot \mathrm{min}^{-1}$ (cases 17, 1 and 18, respectively). The mass loss variation due to the heating rates can be observed in Figure 23 . The reactions occur at lower temperatures in case $17\left(5 \mathrm{~K} \cdot \mathrm{min}^{-1}\right)$. The oxidative reactions in case $18\left(20 \mathrm{~K} \cdot \mathrm{min}^{-1}\right)$ are delayed, and the oxidative reactions become competitive as it can be seen in the Figure 24.

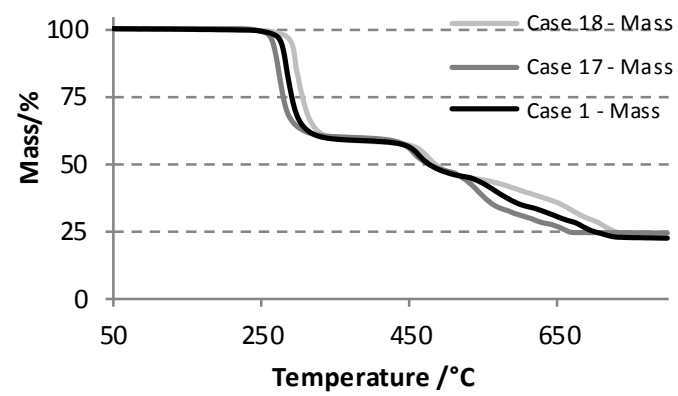

Fig. 23 Heating rate influence on PVC TG curve.

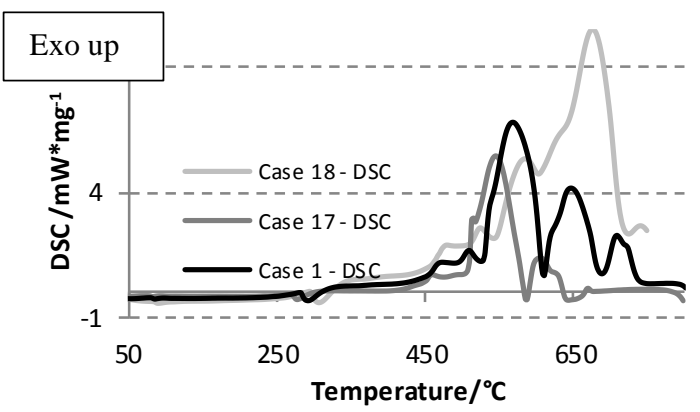

Fig. 24 Heating rate influence on PCV DSC curve.

Figures 25 and 26 represent the derivative of the mass versus time (DTG) and versus temperature (DTG*), respectively. Although in fire science the most used is the mass loss rate (derivative versus time), the derivative versus temperature allows to perform a better comparison of the reactions obtained under STA tests at different heating rates. This is because a particular decomposition reaction should loss the same amount of mass, and although initial and final decomposition temperature were different, the temperature range between them should be the same. When we use temperature to derivate, we are comparing same mass loss divided by same variation of temperature independently of the heating rate, but if we consider time, we will compare same variation of mass divided by different range of time.

This can be seen in the Figures 25 and 26. Although the dehydrochlorination reaction is the same in all cases, it is represented by different height and shape of the DTG curve peak, nevertheless, the dehydrochlorination reaction 
DTG* curve peak has same height and shape independently of the heating rate. The only difference in the curve is the temperatures at with decomposition takes place.

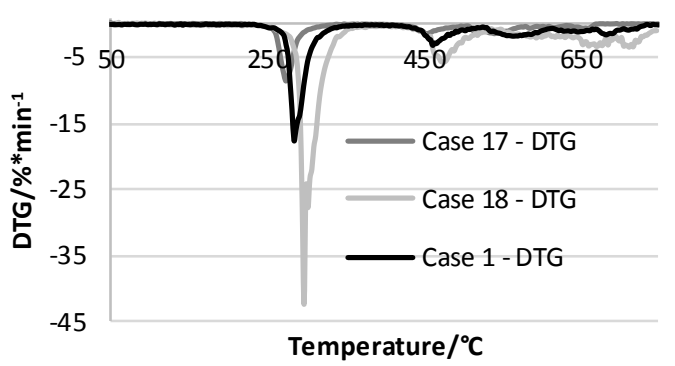

Fig. 25 Heating rate influence on PVC DTG curve.

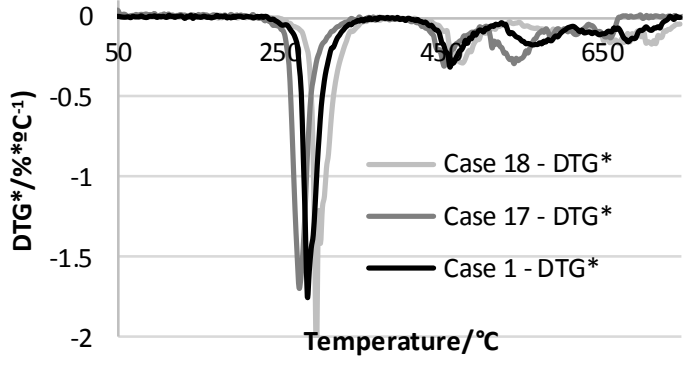

Fig. 26 Heating rate influence on PVC DTG curve.

Figures 27 and 28 show in detail the mass and DTG* of the oxidative reactions of PVC. It can be seen that the first oxidative reaction is the same for all cases (similar DTG* peak), but the rest oxidative reactions changes with the heating rate (different DTG* peaks).

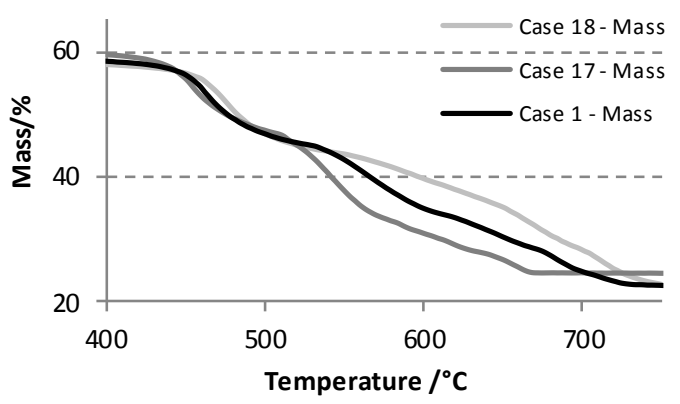

Fig. 27 Detail of heating rate influence on PVC TG curve.

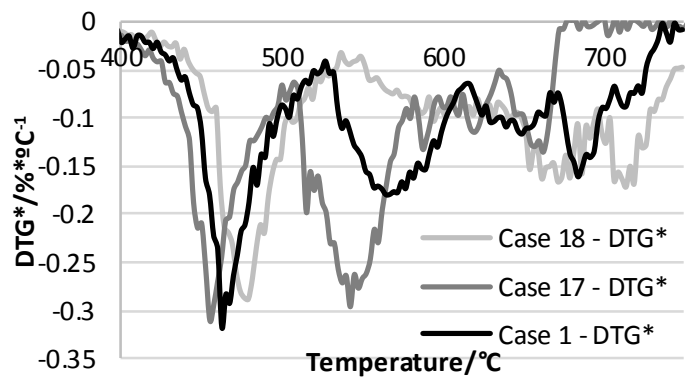

Fig. 28 Detail of heating rate influence on PVC DTG* curve.

The effect of the heating rate on LLDPE decomposition is shown in the figures 29 to 32 , where mass, DSC, DTG and DTG* are represented. Cases 35, 19 and 34 consider heating rates of 5, 10 and $20 \mathrm{~K} \cdot \mathrm{min}^{-1}$, respectively. The decomposition happens at lower temperatures for Case 35 (Figure 29). Case 34 has the higher decomposition temperatures. As in the PVC tests, DTG shows different scale of the peak height, nevertheless comparing DTG* it can be seen that although DTG* shape and peak height are different, which means that different decomposition reaction are taking place, the scale of the peak is similar. 


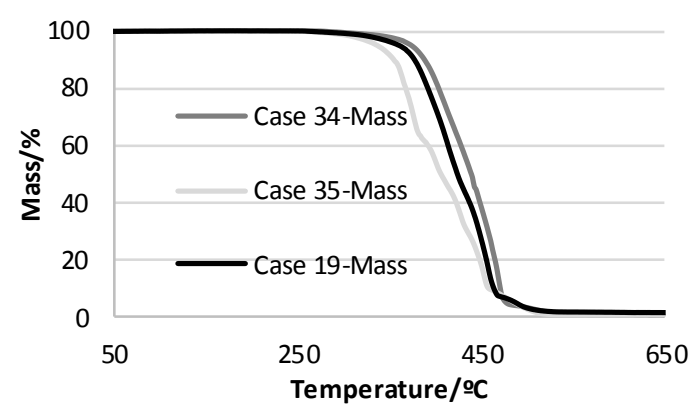

Fig. 29 Heating rate influence on LLDPE TG curve under air atmosphere.

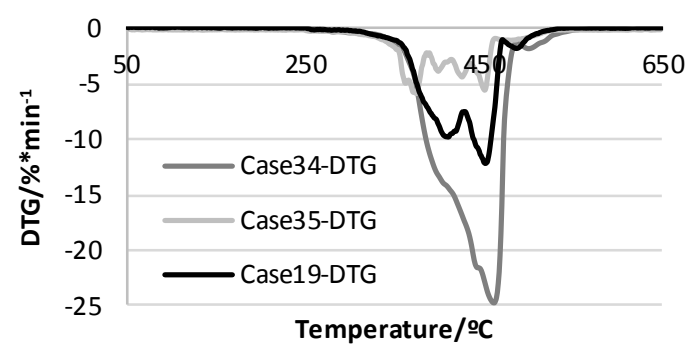

Fig. 31 Heating rate influence on LLDPE DTG curve under air atmosphere.

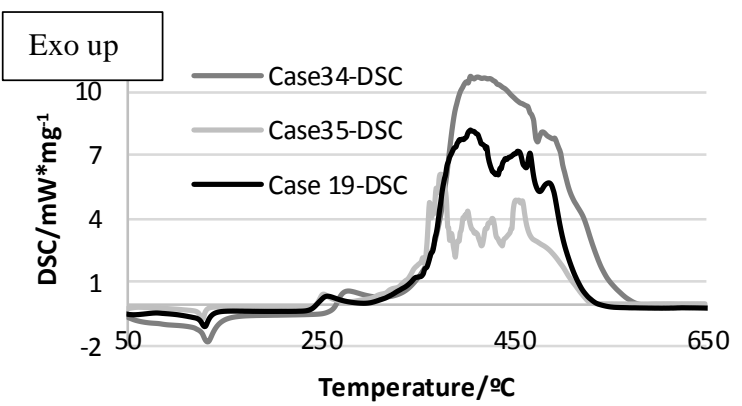

Fig. 30 Heating rate influence on LLDPE DSC curve under air atmosphere.

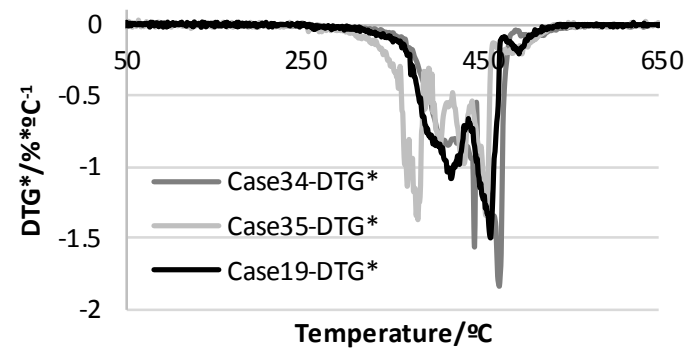

Fig. 32 Heating rate influence on LLDPE DTG* curve under air atmosphere.

To analyze the heating rate influence on LLDPE decomposition under nitrogen atmosphere, it has been selected the heating rates of 2, 5 and $10 \mathrm{~K} \cdot \mathrm{min}^{-1}$ for cases 26,25 and 24 respectively. Figures 33, 34 and 35 show the mass loss, the DTG and the DTG*. It can be seen that the decomposition temperature is higher for the higher heating rate (case 24). Figure 34 shows that the peak of mass loss rate is delayed, but the shape and height of the peaks are completely different, nevertheless comparing the DTG* (Figure 35), it can be seen that the height and the shape of the decomposition peak are the same for all cases. This means that the decomposition reaction of the LLDPE under nitrogen atmosphere is the same independently of the heating rate.

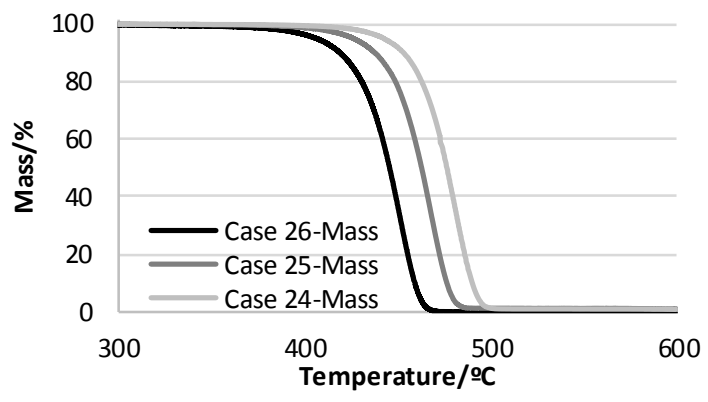

Fig. 33 Heating rate influence on LLDPE TG curve under nitrogen atmosphere. 


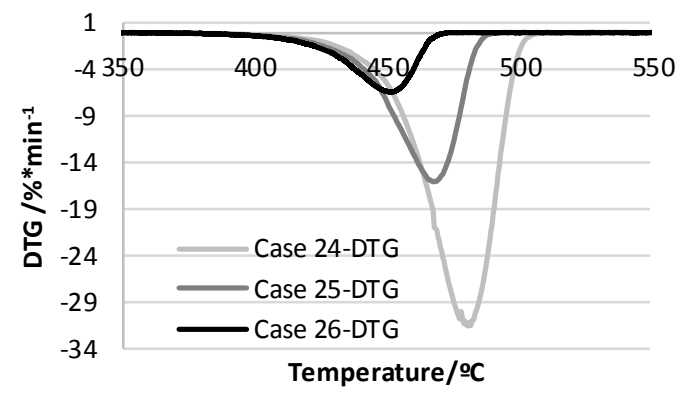

Fig. 34 Heating rate influence on LLDPE DTG curve under nitrogen atmosphere.

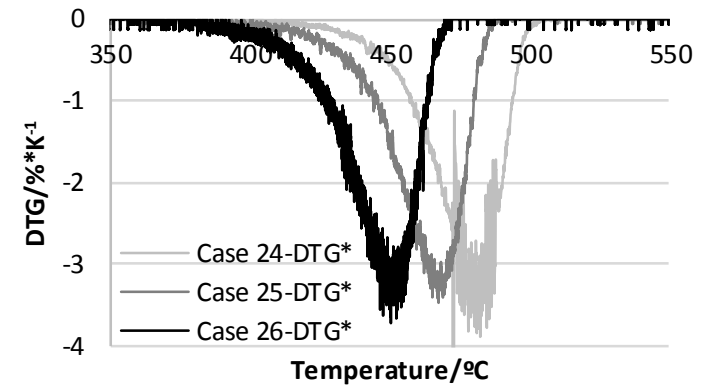

Fig. 35 Heating rate influence on LLDPE DTG* curve under nitrogen atmosphere.

The analysis of the heating rate influence over PVC and LLDPE decomposition shows that the heating rate not only affect the decomposition temperature, but also it can affect to the kind of decomposition reactions. In the study, the reactions most affected by the heating rates are the oxidative ones. This could be related with the gas release rate during decomposition and the amount of gas released that burns close to the sample. As lower is the heating rate as lower is the gas released and more quantity of this gas will burn in the sample increasing the temperature over the sample, and so on the rate of the initials decomposition reactions (as it can be seen in the Figures 27 and 28).

\section{CONCLUSIONS}

This work aims to study how the STA experimental boundary conditions affect thermal decomposition of polymers. A sensitivity analysis of the influence of the boundary conditions in the thermal decomposition of PVC and LLDPE was performed. We can resume the conclusions in the following statements:

- Mass sample variation affects slightly the mass loss rate and energy release of the oxidative thermal decomposition reactions. Results suggested that higher influence of the mass on the thermal decomposition reactions is related with the availability of oxygen by the sample during its decomposition. Although mass samples above $10 \mathrm{mg}$ are recommended by some standards [4-6], mass samples lower than $10 \mathrm{mg}$ might be also recommended to perform experimental tests ensuring a low temperature gradient inside the sample and lower effect on the decomposition of the energy release by the oxidative reactions.

- Gas flow affects the reaction of the gases released in the thermal decomposition of the sample. Better aeration may well improve the oxidative chemical reactions allowing higher amount of heat released. Chemical reactions that not depend on the atmosphere are not influenced by the gas flow.

- The use of a pierced lid under an inert atmosphere avoids the influence of the heat flux rate of radiation when sample and reference have different emissivities and improves the heat distribution in the crucible. The use of pierced lid in an oxidative atmosphere provides a self-generated atmosphere to the sample.

- Oxygen content of the oxidative atmosphere determines oxidative reactions of combustion. Varying oxygen content in STA allows studying under-ventilated combustion reactions.

- The heating rate not only affects the temperature at which decomposition reactions takes place, but also modifies the kind of oxidative reactions during decomposition. This could affect the standard methodologies to obtain kinetic parameters that define the use of several tests performed under different heating rates to reach a decomposition reaction

Although the sensitivity analysis was performed with PVC and LLDPE samples, similar decomposition reactions are highly likely to be affected in the same way for other thermoplastic materials. The previous statements present the qualitative influence of the boundary conditions in the polymers. In order to assess a quantitative influence detailed analysis of each polymer should be done. All in all, every boundary condition studied in the 
present analysis have influence in the thermal decomposition, and they should be well defined to perform the test attending to the desired conditions by the user.

Acknowledgment Authors would like to thank to the Consejo de Seguridad Nuclear for the cooperation and cofinancing the project "Simulation of fires in nuclear power plants" and to CAFESTO Project funded by the Spanish Ministry of Science, Innovation and Universities and the Spanish State Research Agency through Public-Private Partnerships (Retos Colaboración 2017 call, ref RTC-2017-6066-8) co-funded by ERDF under the objective "Strengthening research, technological development and innovation".

\section{REFERENCES}

1. Witkowski A, Stec AA, Hull TR. Chapter 7. Thermal Decomposition of Polymeric Materials. In: SFPE, editor. SFPE Handbook of Fire Protection Engineering, Fifth Edition. 2016. p. 1-3493.

2. Sun $Y$, Gao M, Chai Z, Wang $H$. Thermal behavior of the flexible polyvinyl chloride including montmorillonite modified with iron oxide as flame retardant. J Therm Anal Calorim. 2018;131(1):65-70.

3. Alonso A, Puente E, Lázaro P, Lázaro D, Alvear D. Experimental review of oxygen content at mixing layer in cone calorimeter. J Therm Anal Calorim. 2017;129(2).

4. ASTM Standard E1131 - 08 Standard Test Method for Compositional Analysis by Thermogravimetry. West Conshohocken, PA; 2014.

5. EN ISO 11358-1:2015 Plastics - Thermogravimetry (TG) of Polymers-Part 1: General principles.

6. UNE-EN ISO 11357-1. Plásticos Calorimetría diferencial de barrido (DSC) Parte 1: Principios generales. (ISO 11357-1:2016).

7. Schindler A, Doedt M, Gezgin Ş, Menzel J, Schmölzer S. Identification of polymers by means of DSC, TG, STA and computer-assisted database search. J Therm Anal Calorim. 2017;129(2):833-42.

8. Vyazovkin S, Chrissafis K, Di Lorenzo ML, Koga N, Pijolat M, Roduit B, et al. ICTAC Kinetics Committee recommendations for collecting experimental thermal analysis data for kinetic computations. Thermochim Acta. 2014;590:1-23.

9. Marcilla A, Beltran M. Thermogravimetric kinetic study of poly (viny1 chloride ) pyrolysis. Polym Degrad Stab. 1995;3910(95):219-29.

10. Miranda R, Pakdel H, Roy C, Darmstadt H, Vasile C. Vacuum pyrolysis of PVC I: Kinetic study. Polym Degrad Stab. 1999;66(1):107-25.

11. Morais LC, Maia AAD, Guandique MEG, Rosa AH. Pyrolysis and combustion of sugarcane bagasse. J Therm Anal Calorim. 2017;129(3):1813-22.

12. Wu J, Wang B, Cheng F. Thermal and kinetic characteristics of combustion of coal sludge. J Therm Anal Calorim. 2017;129(3):1899-909.

13. Xu W, Li J, Liu F, Jiang Y, Li Z, Li L. Study on the thermal decomposition kinetics and flammability performance of a flame-retardant leather. J Therm Anal Calorim. 2017;128(2):1107-16.

14. Zong R, Wang Z, Liu N, Hu Y, Liao G. Thermal degradation kinetics of polyethylene and silane-crosslinked polyethylene. J Appl Polym Sci. 2005;98(3):1172-9.

15. Capote J, Alvear D, Abreu O, Lázaro M, Puente E. Modelling pyrolysis of a medium density polyethylene. Int Rev Chem Eng. 2010;2-7.

16. Lázaro D, Puente E, Lázaro M, Alvear D. Characterization of Polyethylene Decomposition Reactions Using the TG Curve. Int Rev Chem Eng. 2014;6(January):77-82.

17. Peterson JD, Vyazovkin S, Wight CA. Kinetics of the thermal and thermo-oxidative degradation of polystyrene, polyethylene and poly(propylene). Macromol Chem Phys. 2001;202(6):775-84.

18. Jiménez A, Iannoni A, Torre L, Kenny JM. Kinetic modeling of the thermal degradation of stabilized PVC plastisols. J Therm Anal Calorim. 2000;61(2):483-91. 
19. Marquis DM, Guillaume E, Camillo A, Rogaume T, Richard F. Existence and uniqueness of solutions of a differential equation system modeling the thermal decomposition of polymer materials. Combust Flame [Internet]. 2013;160(4):818-29. Available from: http://dx.doi.org/10.1016/j.combustflame.2012.12.008

20. Marquis DM, Pavageau M, Guillaume E, Chivas-Joly C. Modelling decomposition and fire behaviour of small samples of a glass-fibre-reinforced polyester/balsa-cored sandwich material. Fire Mater. 2013;37:413-39.

21. Abu-Bakar AS, Moinuddin KAM. Effects of Variation in Heating Rate, Sample Mass and Nitrogen Flow on Chemical Kinetics for Pyrolysis. 18th Australas Fluid Mech Conf. 2012;(December):18-21.

22. Stawski D. The effect of sample weight in thermogravimetric analysis of low viscosity polypropylene in air atmosphere. Polym Test. 2009;28(2):223-5.

23. Luo J, Li Q, Meng A, Long Y, Zhang Y. Combustion characteristics of typical model components in solid waste on a macro-TGA. J Therm Anal Calorim [Internet]. 2018;132(1):553-62. Available from: https://doi.org/10.1007/s10973-017-6909-9

24. ASTM E1269-11(2018), Standard Test Method for Determining Specific Heat Capacity by Differential Scanning Calorimetry, ASTM International, West Conshohocken, PA, 2018, www.astm.org.

25. Wolfinger MG, Rath J, Krammer G, Barontini F, Cozzani V. Influence of the emissivity of the sample on differential scanning calorimetry measurements. Thermochim Acta. 2001;372(1-2):11-8.

26. Posch W. Polyolefins. In: Applied Plastics Engineering Handbook [Internet]. 2011. p. 23-48. Available from: http://linkinghub.elsevier.com/retrieve/pii/B9781437735147100030 